\title{
Aprendizaje de la historia clínica con pacientes simulados en el grado de Medicina
}

\author{
M. Cristina Rodríguez-Díez, Juan J. Beunza, Cristina López-Del Burgo, Omar Hyder, M. Pilar Civeira-Murillo, \\ Nieves Díez
}

Objetivos. La utilización de la simulación es habitual en medicina para mejorar el conocimiento y habilidades de competencias. Se pueden utilizar pacientes virtuales, simuladores y pacientes simulados. Nosotros planteamos que alumnos de quinto y sexto curso actúen como pacientes simulados para que sus compañeros de primero aprendan la anamnesis de la historia clínica.

Sujetos y métodos. Un total de 207 alumnos de primer curso de grado aprendieron a realizar la historia clínica en una sala de urgencias del centro de simulación entrevistando a pacientes simulados, que eran sus compañeros de quinto y sexto curso. Se evaluaron las historias clínicas realizadas, y para conocer el grado de satisfacción de los estudiantes se pidió que cumplimentasen un cuestionario anónimo tanto a los alumnos de primer curso como a los de cursos superiores.

Resultados. La valoración de la calidad de las historias clínicas fue de 8,2 sobre 10. Esta metodología ha sido muy bien valorada. A la pregunta '¿el entrenamiento con pacientes simulados ayuda al aprendizaje para la realización de la historia clínica?', los alumnos de primero puntuaron 9, y los de quinto y sexto, 9,2. Al ítem '¿trabajar con pacientes simulados mejora sus habilidades de comunicación?', ambos grupos puntuaron 8,6. Y para '¿es adecuado el aprendizaje de la historia clínica mediante pacientes simulados previo a su aprendizaje con pacientes reales?', ambos grupos puntuaron 9,3 . La valoración de la experiencia como paciente simulado fue de 9,3.

Conclusión. El aprendizaje de la historia clínica en primer curso del grado de Medicina mediante pacientes simulados resulta beneficioso para los alumnos. Un contacto temprano con la clínica a partir del aprendizaje basado en la simulación podría mejorar la formación de los estudiantes.

Palabras clave. Educación de grado. Estudiantes de medicina. Paciente simulado.

Learning to take medical histories through patients simulation in undergraduate Medical School students

Aims. Simulation techniques are commonly used in medical education to improve the acquisition of knowledge, abilities and competencies. Several methods have been proposed: virtual patients, high fidelity devices and standard patients. We propose the use of 5th-6th year Medical School students acting as patients when teaching history taking to their 1st year colleagues.

Subjects and methods. A total of 207 students from 1st year Medical School underwent training in history taking at the Simulation Center, with senior students acting as actors. The quality of the written medical records was evaluated by two medical doctors. The satisfaction of all students involved in the course was evaluated through an anonymous voluntary questionnaire.

Results. The average score of the written medical histories was 8.2/10, more than satisfactory for our goals. Students' satisfaction rate was high. Mean score on questions inquiring the usefulness of patient simulation in learning how to perform a clinical history was $9 / 10$ and $9.2 / 10$ for first and fifth-sixth year students respectively. Questions on improvement of communication skills scored 8.6/10 and 8.6/10 respectively. The fruitfulness of training with simulated patients before practicing with real patients was $9.3 / 10$ and $9.3 / 10$ respectively. Finally, the assessment of the whole course with simulated patients was of 9.3/10.

Conclusion. Learning history taking in first year Medical School with simulated patients acted by senior students was beneficial and user-friendly for both students and actors. An early contact with the clinical practice through simulated patients could improve performance and safety.

Key words. Medical School. Simulated patients. Undergraduate education.

Departamento de Fisiología Humana (M.C. Rodríguez-Díez, N. Díez); Departamento de Medicina Preventiva y Salud Pública (J.J. Beunza, C. LópezDel Burgo); Unidad de Educación Médica (J.J. Beunza, N. Díez); Facultad de Medicina; Universidad de Navarra. Departamento de Medicina Interna; Clínica Universidad de Navarra (M.P. Civeira-Murillo); Pamplona, Navarra, España. Departamento de Obstetricia y Ginecología; Rawalpindi Medical College (0. Hyder); Rawalpindi, Pakistán.

Correspondencia:

Dra. M. Cristina Rodríguez Díez. Departamento de Fisiología Humana. Facultad de Medicina; Universidad de Navarra. Irunlarrea, 1. E-31008 Pamplona (Navarra).

E-mail:

cristinard@unav.es

Conflicto de intereses: No declarado.

Competing interests: None declared.

Los resultados de este trabajo fueron aceptados como comunicación oral en el congreso de la Sociedad Europea para la Simulación Aplicada a la Medicina (SESAM), celebrado en Granada, 1-4 de junio de 2011.

(C) 2012 Educación Médica 


\section{Introducción}

El objetivo de la declaración de Bolonia es disponer de un Espacio Europeo de Educación Superior, lo que supone para las facultades de medicina adaptar los nuevos planes docentes. Los estudios de medicina tienen un gran componente práctico e incluyen en algunas asignaturas las técnicas de simulación con el objetivo de mejorar habilidades de comunicación, trabajo en equipo y competencias específicas. En los planes de estudios anteriores a Bolonia, los alumnos de primeros cursos se formaban en conocimientos teóricos básicos y no era hasta los cursos superiores cuando iniciaban su contacto con el paciente. Esta relación tardía con el medio sanitario daba lugar a que a algunos estudiantes les costase adquirir un razonamiento clínico adecuado. Por tanto, planificar el currículo académico de manera que el alumno tenga contacto con el paciente desde primer curso podría contribuir a mejorar esta deficiencia [1].

Se han propuesto diferentes metodologías para mejorar el aprendizaje de los estudiantes de medicina. Entre las más destacadas figuran el aprendizaje basado en problemas [2], la evaluación clínica objetiva estructurada [3], la utilización de portafolios $[4,5]$ y la simulación médica [6,7]. Por tanto, la simulación en medicina no es novedosa en otros países y se ha empleado en las últimas décadas con resultados satisfactorios [8,9]. En España, las facultades de medicina están iniciando el desarrollo de esta metodología de enseñanza. Una gran ventaja de esta técnica es que permite a los estudiantes trabajar en escenarios simulados, lo que implica numerosos beneficios y escasos riesgos. Las modernas técnicas de simulación pueden resumirse, brevemente, en modelos informáticos (pacientes virtuales), maniquíes (modelos de simuladores de tamaño a escala real, simuladores para técnicas concretas) y pacientes simulados.

Barrows [10] define paciente simulado/estandarizado como 'una persona que ha sido preparada cuidadosamente para actuar como paciente de manera que no pueda ser detectado por el médico'. Asimismo, el modo en que el paciente simulado interactúa con los estudiantes puede ser diferente: en algunos casos lo hacen mínimamente, mientras que en otros interactúan más [11]. Teniendo en cuenta que la simulación con actores entrenados es una metodología costosa, algunos investigadores han trabajado con estudiantes que simulaban ser pacientes [7].

El objetivo de este trabajo ha sido evaluar y describir los beneficios de la simulación en los estudiantes de primer curso del grado de Medicina me- diante el aprendizaje de la entrevista clínica teniendo como pacientes simulados a compañeros de cursos superiores. En la asignatura de 'Iniciación a la Clínica, el alumno adquiriere los conocimientos, hábitos y destrezas básicas realizando la anamnesis a los pacientes simulados de manera que en la redacción de la historia clínica se reflejen los datos de filiación, el motivo de la consulta, la ordenación cronológica, la descripción de los síntomas, los antecedentes personales y familiares y los hábitos. Desde tercero hasta sexto curso, en las asignaturas de 'Clínica Práctica', los alumnos realizan las pasantías en los hospitales y en los centros de atención primaria, donde desarrollan y perfeccionan las habilidades de comunicación, redacción y obtención de la anamnesis con los pacientes reales.

\section{Sujetos y métodos}

Un total de 207 estudiantes se matricularon en la asignatura de 'Iniciación a la Clínica' de primero del grado de Medicina durante el curso académico 2010-2011. El programa de la asignatura se divide en seis unidades y la unidad 1 comprende la anamnesis de la historia clínica. El contenido teórico se impartió en tres clases teóricas y se trabajó en cuatro talleres de dos horas de duración cada uno. Cada taller hacía especial énfasis en una parte de la anamnesis (historia actual de la enfermedad, antecedentes personales y familiares, hábitos y anamnesis por aparatos). En cada taller, los alumnos redactaron las historias clínicas obtenidas de la entrevista a los pacientes simulados.

Los pacientes simulados eran alumnos de quinto y sexto curso de la misma facultad a los que se invitó a participar a través de un correo electrónico y también se informó directamente en el aula. La participación de estos estudiantes fue voluntaria y obtuvieron créditos de libre elección. Los pacientes simulados fueron entrenados previamente por las profesoras responsables de la asignatura para cada caso que debían interpretar. Se mantuvieron dos reuniones con los pacientes simulados; en la primera de ellas se explicó a los alumnos los objetivos de su trabajo y se les entregaron por escrito los casos clínicos que debían trabajarse en cada taller. En la segunda reunión, una de las profesoras actuó como paciente simulado y el alumno era quien realizaba la anamnesis de manera que se hacía especial hincapié en cómo debían ser las respuestas.

El desarrollo de los escenarios simulados tuvo lugar en una sala del centro de simulación de la Facultad de Medicina que reproduce una sala de ur- 
gencias. Esta sala disponía de todo el material necesario para la grabación de los escenarios clínicos (sistema de integración audiovisual, altavoces, micrófonos, cámaras de video y monitores). Desde las salas de los talleres, los demás alumnos del grupo visualizaron en tiempo real la entrevista, que además se grababa. Posteriormente, el alumno de primero y el paciente simulado se incorporaron a la sala donde tuvo lugar el análisis de la entrevista clínica, que era dirigida por el médico responsable del taller. Cuando era necesario reforzar algunos puntos se proyectaba de nuevo la grabación (Figura).

\section{Evaluación de las historias clínicas}

Cada alumno redactó varias historias clínicas y en el último taller se recogió una de ellas para su valoración. La profesora responsable de la asignatura y directora del centro de simulación evaluó cada historia clínica de acuerdo con una lista de 10 ítems que se considera que definen una buena historia clínica. Así se valoró el orden, contenido e inclusión de todos las partes de la historia clínica: enfermedad actual, antecedentes personales y familiares, hábitos y anamnesis por sistemas, así como la expresión y uso apropiado de terminología médica.

\section{Evaluación de los alumnos}

Al finalizar la unidad se solicitó a todos los alumnos de primer curso que cumplimentaran un cuestionario anónimo de satisfacción con ocho ítems (Tabla I). Asimismo, a los alumnos de quinto y sexto curso se les pidió que respondiesen a otro cuestionario anónimo de satisfacción con 17 ítems (Tabla II). Las preguntas se referían a competencias relacionadas con trabajo en equipo, comunicación, motivación, razonamiento e integración de conocimientos. Todas las cuestiones se respondían en una escala de 1 (puntuación más baja) a 10 (puntuación más alta).

\section{Análisis de datos}

El análisis estadístico se realizó con el programa SPSS v. 15.0.

\section{Resultados}

\section{Evaluación de la calidad de las historias clínicas realizadas por los alumnos de primer curso}

La historia clínica realizada por los alumnos se corrigió de acuerdo con los criterios establecidos pre-
Figura. Alumnos de primero y sexto curso de medicina.

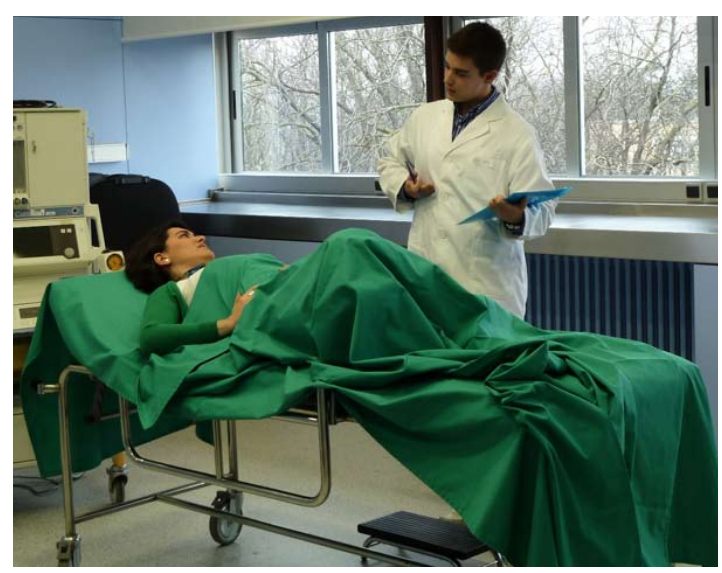

viamente. Sobre 10 puntos como máximo, la puntuación media obtenida fue de 8,2 $\pm 1,1$.

\section{Evaluación de la actividad con pacientes simulados por parte de los alumnos de primer curso}

Un total de 207 alumnos estaban matriculados en primer curso. De ellos, 205 (99\%) respondieron al cuestionario. La edad media de los alumnos era de 18,3 años. La distribución por sexo fue de $127 \mathrm{mu}$ jeres (62\%) y 78 hombres (38\%).

En la tabla I se muestra la valoración a cada pregunta. En siete de los ocho ítems, la valoración fue igual o superior a 8. A la cuestión '¿cree que trabajar con pacientes simulados que fuesen actores profesionales sería mejor que la realizada con compañeros de cursos superiores?', la puntuación fue de 3,6.

\section{Valoración de los pacientes simulados actores (alumnos de quinto y sexto curso)}

Las encuestas de satisfacción fueron respondidas por nueve de los diez alumnos que participaron como pacientes simulados. La edad media de los alumnos fue de 22,6 años. La distribución por sexo fue de siete mujeres $(77,8 \%)$ y dos hombres $(22,2 \%)$.

La tabla II muestra la valoración a cada ítem planteado. Así, en 15 de 17 obtuvieron una valoración igual o superior a 8 . A la pregunta ' $¿$ le ha servido para mejorar su propio conocimiento de algunas patologías?', la valoración fue de 7,3, y a la pregunta ¿'le parecería mejor que fuesen actores de teatro los pacientes simulados?' respondieron con una pun- 
Tabla I. Puntuaciones de los alumnos de primer grado $(n=205)$ sobre la utilización de alumnos simuladores de pacientes para el aprendizaje de la historia clínica.

\begin{tabular}{lc}
\hline & Media \pm DE \\
\hline ¿Cree que el entrenamiento con pacientes simulados ayuda al aprendizaje para la realización de la historia clínica? & $9,0 \pm 0,97$ \\
\hline ¿Cree que trabajar con pacientes simulados ayuda a la integración y razonamiento de los conocimientos teóricos? & $8,8 \pm 1,15$ \\
\hline ¿Cree que trabajar con pacientes simulados ayuda a mejorar sus habilidades de comunicación? & $8,6 \pm 1,41$ \\
\hline ¿Cree que las historias clínicas fueron adecuadas al nivel de sus conocimientos? & $8,1 \pm 1,43$ \\
\hline ¿Le resulta motivador ver participar a los alumnos de cursos superiores como pacientes simulados? & $8,3 \pm 1,67$ \\
\hline \begin{tabular}{l} 
¿Le parece que debe incluirse esta metodología de aprendizaje en el plan de estudios de cursos superiores? \\
\hline ¿Le parece adecuado el aprendizaje de la historia clínica mediante pacientes simulados previo a su aprendizaje \\
con pacientes reales?
\end{tabular} & $8,2 \pm 1,83$ \\
\hline ¿Le parecería mejor que fuesen actores de teatro los pacientes simulados? & $9,3 \pm 1,01$
\end{tabular}

Cada pregunta se valoró de 1 (puntuación mínima) a 10 (puntuación máxima). DE: desviación estándar.

tuación de 4,3. Por último, la valoración de su experiencia como paciente simulado fue de 9,3.

\section{Discusión}

Este es el tercer curso académico en que alumnos de cursos superiores de la Facultad de Medicina de la Universidad de Navarra colaboran en la docencia de la historia clínica a los alumnos de primer año. Respecto a los cursos previos hemos añadido como mejora que la historia clínica se realiza en un escenario que reproduce una sala de urgencias del centro de simulación, lo que permite un mayor realismo.

Según Miller, las competencias profesionales se evalúan en cuatro niveles: saber, saber cómo, demostrar y hacer [12]. Siguiendo esta escala del aprendizaje, los alumnos adquieren los conocimientos básicos de esta competencia a partir de las clases teóricas, pero es entrevistando a los pacientes simulados cuando demuestran y realizan la adquisición de la habilidad. Asimismo, mediante la simulación facilitamos que los alumnos puedan repetir los casos clínicos tantas veces como sea necesario, algo que no puede llevarse a cabo con pacientes reales. Es cierto que para aprender a realizar la historia clínica siempre es necesario el contacto con el 'paciente real'. Sin embargo, consideramos que si previamente los estudiantes entrevistan a pacientes simulados en un ambiente seguro, cercano y con sus compañeros y profesores, de los que recibirán comentarios y reflexiones, ello favorecerá que cuando se enfrenten a los pacientes reales conozcan mejor la estructura de la entrevista clínica y demuestren mayores habilidades de comunicación. Esto se ha reflejado en la evolución de los talleres y en la redacción de la historia clínica. Hemos incorporado esta metodología de aprendizaje y ha resultado muy satisfactorio para ambos grupos de estudiantes.

Con los pacientes simulados se pueden trabajar, en asignaturas de últimos cursos, gran variedad de patologías, enfermedades raras o situaciones críticas que son poco habituales en la vida real [13]. Para nuestra asignatura, 'Iniciación a la Clínica', ha supuesto grandes ventajas. En primer lugar, desde primero de grado los alumnos comienzan a practicar la técnica de la entrevista clínica y a saber relacionarse con los pacientes, en este momento simulados. En segundo término, para los estudiantes de primer curso, relacionarse con los estudiantes mayores favorece la motivación y colaboración y, por otra parte, para los estudiantes de cursos superiores, actuar como pacientes simulados requiere conocer en detalle las patologías que van a interpretar. Además, la experiencia de sentirse pacientes puede ayudarlos a conocer mejor el punto de vista de los pacientes reales. Por último, otra ventaja es que no supone incremento en los costes de docencia frente a una posible utilización de actores profesionales. 
Tabla II. Puntuaciones de los alumnos de los cursos 5 y 6으 $(n=9)$ sobre la utilización de alumnos simuladores de pacientes para el aprendizaje de la historia clínica.

\begin{tabular}{|c|c|}
\hline & Media $\pm \mathrm{DE}$ \\
\hline ¿Cree que el entrenamiento con pacientes simulados ayuda al aprendizaje para la realización de la historia clínica? & $9,2 \pm 1,30$ \\
\hline ¿Le parece adecuado iniciar el aprendizaje de la historia clínica en primer curso de medicina? & $8,7 \pm 1,64$ \\
\hline ¿Cree que trabajar con pacientes simulados ayuda a la integración y razonamiento de los conocimientos teóricos? & $8,5 \pm 1,23$ \\
\hline ¿Cree que trabajar con pacientes simulados ayuda a mejorar las habilidades de comunicación? & $8,6 \pm 1,93$ \\
\hline ¿Cree que las historias clínicas fueron adecuadas al nivel de conocimientos de los alumnos de primer curso? & $8,1 \pm 1,26$ \\
\hline ¿Cree que los alumnos de primero mostraban interés por los talleres? & $8,8 \pm 1,05$ \\
\hline ¿Cree que es motivador para los alumnos de primero ver participar a sus compañeros de cursos superiores como pacientes simulados? & $9,1 \pm 1,05$ \\
\hline ¿Considera que un alumno de quinto o sexto curso tiene capacidad suficiente para actuar como paciente simulado? & $9,7 \pm 0,40$ \\
\hline ¿Cree que recibió información suficiente sobre cómo debía ser su participación? & $9,0 \pm 1,30$ \\
\hline ¿Se sentía cómodo en la actuación como paciente simulado? & $9,4 \pm 0,70$ \\
\hline ¿Le ha servido para mejorar su propio conocimiento de algunas patologías? & $7,3 \pm 1,70$ \\
\hline ¿Le hubiese gustado que en su plan de formación se trabajase con pacientes simulados en el aprendizaje de la historia clínica? & $9,7 \pm 0,44$ \\
\hline ¿Cree que los talleres estaban bien organizados? & $9,2 \pm 0,66$ \\
\hline ¿Le parece que debe incluirse esta metodología de aprendizaje en el plan de estudios de cursos superiores? & $9,0 \pm 1,22$ \\
\hline $\begin{array}{l}\text { ¿Le parece adecuado el aprendizaje de la historia clínica mediante pacientes simulados previo a su aprendizaje } \\
\text { con pacientes reales? }\end{array}$ & $9,3 \pm 0,70$ \\
\hline ¿Le parecería mejor que fuesen actores de teatro los pacientes simulados? & $4,3 \pm 3,60$ \\
\hline Valore su experiencia como paciente simulado & $9,3 \pm 0,70$ \\
\hline
\end{tabular}

Del análisis de nuestro trabajo tiene gran relevancia la sencillez y viabilidad para llevarlo a cabo. Creemos que se puede aplicar fácilmente en las facultades de medicina y que aporta grandes beneficios para los estudiantes de primeros y últimos cursos, mejorando sus habilidades de comunicación, trabajo en equipo y empatía.

Por otra parte, esta metodología de aprendizaje forma parte de la adaptación del currículo en el proceso de Bolonia, y los estudiantes continúan mejorando estas habilidades en cursos superiores mediante las asignaturas de 'Preclínica' y 'Clínica Práctica'.

Por último, nuestro estudio tiene limitaciones. En primer lugar, la evaluación de las historias clínicas se realizó con una escala de valoración no vali- dada. Además, al no disponer de un grupo control, no hemos podido evaluar el valor añadido del uso de pacientes simulados frente a no emplearlos.

En conclusión, el aprendizaje de la historia clínica para los alumnos de primer curso del grado de Medicina, mediante la actuación de pacientes simulados realizada por sus compañeros de quinto y sexto curso, es práctico, eficiente y económico, y favorece la docencia en un ambiente cercano y seguro para el alumno.

El inicio temprano del aprendizaje de esta habilidad mediante la simulación creemos que favorece el proceso de aprendizaje y, a largo plazo, podría repercutir en una mejora de la calidad asistencial y seguridad del paciente. 
Bibliografía

1. Palés JL. Planning a curriculum. Educ Med 2006; 9: 59-65.

2. Barrows HS. A taxonomy of problem-based learning methods. Med Educ 1986; 20: 481-6.

3. Harden RM, Stevenson M, Downie WW, Wilson GM. Assessment of clinical competence using objective structured examination. Br Med J 1975; 1: 447-51.

4. Ross S, Maclachlan A, Cleland J. Students' attitudes toward the introduction of a personal and professional development portfolio: potential barriers and facilitators. BMC Med Educ 2009; 9: 69.

5. Buckey S, Coleman J, Davison I, Khan KS J, Zamora J, Malick S, et al. The educational effects of portfolios on undergraduate student learning: a best evidence medical education (BEME) systematic review. Med Teach 2009; 31: 279-81.

6. Weller JM. Simulation in undergraduate medical education: bridging the gap between theory and practice. Med Educ 2004; 38: 32-8.

7. Unalan PC, Uzuner A, Cifcili SY, Akman M, Hancioglu S,
Thulesius HO. Using theatre in education in a traditional lecture oriented medical curriculum. BMC Med Educ 2009; 69: 73.

8. Mavis B, Turner J, Lovell K, Wagner D. Faculty, students, and actors as standardized patients: expanding opportunities for performance assessment. Teach Learn Med 2006; 18: 130-6.

9. Grazzia R, Visconti A, Balager E, Martínez J. Uso de simuladores en ginecología y obstetricia. Experiencia en la enseñanza de pregrado. Educ Med 2006; 8: 229-33.

10. Barrows HS. An overview of the uses of standardized patients for teaching and evaluating clinical skills. Acad Med 1998; 68: 443-51.

11. Collins JP, Harden RM. AMEE Medical Education Guide no 13: real patient, simulated patients and simulators in clinical examination. Med Teach 1998; 20: 508-21.

12. Miller GE. The assessment of clinical skills, competence, performance. Acad Med 1990; 65: S63-7.

13. Cleland JA, Abe K, Rethans JJ. AMEE Medical Education Guide no. 42: the use of simulates patients in medical education. Med Teach 2009; 31: 477-86. 